\title{
Exact Diagonalization Approach for Atomistic Calculation of Piezoelectric Effects in Semiconductor Quantum Dots
}

\author{
M. ŚWiderski AND M. ZiElińSKI* \\ Institute of Physics, Faculty of Physics, Astronomy and Informatics, Nicolaus Copernicus University, \\ Grudziądzka 5, 87-100 Toruń, Poland
}

\begin{abstract}
We present an exact diagonalization approach for atomistic calculation of excitonic properties of semiconductor nanostructures under piezoelectric field. The method allows for efficient treatment of both single particle and many-body states at a small computational cost and results in a very good agreement with the full diagonalization treatment. We illustrate our approach by analyzing the effect of a piezoelectric field on a spectra of a self-assembled InAs/GaAs lens-shaped quantum dot. We study the influence of linear and quadratic piezoelectric terms on the quantum dot electronic structure and importantly we found that the non-linear, density functional based theory of piezoelectricity produces results very similar to those obtained by a well-established linear approach utilizing empirical parameters.
\end{abstract}

DOI: 10.12693/APhysPolA.129.A-79

PACS: 73.21.La

\section{Introduction}

Semiconductor quantum dots such as selfassembled $[1,2]$ and nanowire quantum dots [3] constitute an important platform for solid-state based devices aiming for applications in quantum information and cryptography [4-6]. One of the key advantages of these nanosystems is that their spectral properties can be tailored by an adjustment of growth conditions, and also by a post-growth application of external fields [7-11]. In particular, the external electric field allows for all-electric control of quantum dot properties such as the excitonic fine structure splitting [12, 13]. A typical characteristic feature of the epitaxially grown nanosystems is the substantial strain field inherently built into the system due to the lattice mismatch of the quantum dot and the surrounding barrier. In the electronic structure calculations the hydrostatic and biaxial strains are naturally incorporated to the Hamiltonian via corresponding deformation potentials [14]. Furthermore, the shear (off-diagonal) component of strain leads to the emergence of local piezoelectric charges in the vicinity of the quantum dot-surrounding material interface [15]. The local piezoelectric charge gives then rise to a global (non-local) piezoelectric field, which is incorporated to the Hamiltonian as an additive potential term. From the computational point of view the piezoelectric field is thus accounted for as any other (external) electric field.

In this work we utilize this, and propose an approach in which we perform computationally efficient (exact diagonalization) calculation of the piezoelectricity. Next, we compare the exact diagonalization approach with the full diagonalization treatment and find a very good

*corresponding author; e-mail: mzielin@fizyka.umk.pl agreement. Moreover, we note that our scheme is not limited to the piezoelectric field and in principle can be utilized for any external electric field of relatively small amplitude. For example, we have successfully applied the same methodology to study effect of external electric field on the excitonic fine structure of $\mathrm{InAs} / \mathrm{InP}$ double nanowire quantum dots (quantum molecules). Results of these calculations will be discussed elsewhere.

Another key theoretical issue is related to a way in which the piezoelectric charge is calculated from the shear strain components. A well-established [16] piezoelectricity theory applied to quantum dots assumes a linear dependence between the shear strain $\epsilon_{j k}\left(\eta_{j}\right.$ in Voigt's notation), and the piezoelectric vector $\boldsymbol{P}$, whereas the linear $e_{i j k}\left(e_{i j}\right)$ coefficients (tensors) are taken as empirical constants

$$
P_{i}=e_{i j k} \epsilon_{j k} \equiv e_{i j} \eta_{j} .
$$

The piezoelectric charge is then calculated as $\rho_{p}=$ $-\operatorname{div} \boldsymbol{P}$. For the zinc-blende lattice symmetry, the equation for $\boldsymbol{P}$ reduces to a particularly simple form

$$
\boldsymbol{P}=e_{14}(\boldsymbol{r})\left(\epsilon_{y z}+\epsilon_{z y}, \epsilon_{x z}+\epsilon_{z x}, \epsilon_{x y}+\epsilon_{y x}\right),
$$

where $e_{14}$ is the piezoelectric modulus, typically obtained from the experiment. It should be noted that first calculations for self-assembled quantum dots using this approach were performed nearly two decades ago [16]. In recent years Bester, Zunger, and coworkers [17-19] proposed a non-linear approach using the linear $e_{i j}$ and quadratic $B_{i j k}$ piezoelectric tensors derived from the density functional theory (DFT):

$$
P_{i}=e_{i j} \eta_{j}+\frac{1}{2} \sum_{j k} B_{i j k} \eta_{j} \eta_{k} .
$$

Surprisingly it was found, by applying the above theory to quantum dot calculations, that the quadratic term has a similar magnitude as the linear term and that two terms tend to cancel each other $[17,18]$. Further, these authors concluded [17] that "In fact, neglecting the piezoelectric 
effect is a better approximation than using only the linear term". In this work we show that the above conclusion is an overstatement: we found that the linear theory with empirical (experimental) coefficients gives very similar results to a non-linear approach with the DFT derived parameters. Hence, the important role of parametrization.

\section{Method}

To account for material (e.g. InAs/GaAs) lattice mismatch we first calculate strain relaxed positions using the atomistic valence force field (VFF) approach of Keating [20]. Next, we use atomic positions of the relaxed lattice to calculate strain field [14], which are further utilized to calculate piezoelectric vectors and charges according to Eq. (1) (or Eq. (3) for the non-linear piezoelectricity). The resulting piezoelectric potential is calculated from the Poisson equation [15]:

$$
\Delta V_{p}(\boldsymbol{r})=\frac{\rho_{p}(\boldsymbol{r})}{\epsilon_{0} \epsilon_{s}(\boldsymbol{r})}-\frac{1}{\epsilon_{s}(\boldsymbol{r})} \nabla V_{p}(\boldsymbol{r}) \cdot \nabla \epsilon_{s}(\boldsymbol{r}) .
$$

The piezoelectric potential is then added to the tightbinding Hamiltonian as an additive term

$$
\hat{H}=\hat{H}^{0}+\hat{V}_{p},
$$

where in this work $\hat{H}^{0}$ is the $s p^{3} d^{5} s^{*}$ tight-binding [21] Hamiltonian with the nearest neighbors coupling, spinorbit effect [22] and $d$-orbitals included. For a million atom quantum dot the dimension of the Hamiltonian exceeds $10^{7}$. Hamiltonian (5) is then (partially) diagonalized with only several lowest electron and hole states obtained in the calculation. These states are then used in the next step: the many-body calculation [23]. For a general case $\left(\hat{H}=\hat{H}^{0}+\hat{V}\right)$, such as external electric field of different magnitudes and directions, this computationally intensive procedure needs to be repeated many times for each field value.

An alternative approach, proposed in this work, is to perform diagonalization of $\hat{H}^{0}$ only once and then treat $\hat{V}_{p}$ (or $\hat{V}$ in general case) as a perturbation, solved by the exact diagonalization method. In such a case we start with diagonalization of $\hat{H}^{0}$ :

$$
\hat{H}^{0}\left|\Psi_{i}^{0}\right\rangle=E^{i}\left|\Psi_{i}^{0}\right\rangle \text {. }
$$

We find several lowest $\left(N_{\mathrm{el}}\right)$ electron and hole $\left(N_{\mathrm{ho}}\right)$ states, which we denote with lower-case indices

$$
\hat{H}^{0}\left|\Psi_{i, \mathrm{el}}^{0}\right\rangle=E_{\mathrm{el}}^{i}\left|\Psi_{i, \mathrm{el}}^{0}\right\rangle \quad i=1, N_{\mathrm{el}},
$$

and

$$
\hat{H}^{0}\left|\Psi_{i, \mathrm{ho}}^{0}\right\rangle=E_{\mathrm{ho}}^{i}\left|\Psi_{i, \mathrm{ho}}^{0}\right\rangle \quad i=1, N_{\mathrm{ho}} .
$$

Next, we expand Hamiltonian (5) in the basis of $\hat{H}^{0}$ eigenstates. We observe that for the large effective single particle gap $\left(E_{\mathrm{el}}^{1}-E_{\mathrm{ho}}^{1}\right)$ and low-magnitude fields, such as the piezoelectric field, we can perform this basis expansion separately for the electron and the hole subspaces

$$
\begin{aligned}
& \hat{H_{i j}^{\mathrm{el}}}=\left\langle\Psi_{i, \mathrm{el}}^{0}|\hat{H}| \Psi_{j, \mathrm{el}}^{0}\right\rangle=E_{\mathrm{el}}^{i} \delta_{i j}+\left\langle\Psi_{i, \mathrm{el}}^{0}|\hat{V}| \Psi_{j, \mathrm{el}}^{0}\right\rangle, \\
& \text { and } \\
& \hat{H_{i j}^{\mathrm{ho}}}=\left\langle\Psi_{i, \mathrm{ho}}^{0}|\hat{H}| \Psi_{j, \mathrm{ho}}^{0}\right\rangle=E_{\mathrm{ho}}^{i} \delta_{i j}+\left\langle\Psi_{i, \mathrm{ho}}^{0}|\hat{V}| \Psi_{j, \mathrm{ho}}^{0}\right\rangle
\end{aligned}
$$

To calculate electric field matrix elements, we note that the tight-binding wave functions obtained from (7) and (8) are given as a linear combinations of atomic orbitals (LCAO):

$$
\left|\Psi_{i}\right\rangle=\sum_{\alpha, \boldsymbol{R}} c_{\alpha, \boldsymbol{R}}^{i}\left|\Phi_{\alpha, \boldsymbol{R}}\right\rangle,
$$

where $c_{\alpha, \boldsymbol{R}}^{i}$ are the basis expansion coefficients in the atomic ( $\boldsymbol{R}$ centered) basis $\left|\Phi_{\alpha, \boldsymbol{R}}\right\rangle$. The $V$ matrix elements (in a unperturbed basis) are then given as

$$
\left\langle\Psi_{i, \mathrm{el}}^{0}|\hat{V}| \Psi_{j, \mathrm{el}}^{0}\right\rangle=\sum_{\alpha, \boldsymbol{R}}\left|c_{\alpha, \boldsymbol{R}}\right|^{2} V(\boldsymbol{R}),
$$

where we have utilized atomic basis orthogonality [24]. Once constructed, Hamiltonians (9) and (10) are diagonalized, with electron and hole states given as linear combinations of $\hat{H}^{0}$ electron and hole eigenstates

$$
\begin{gathered}
\left|\Psi_{i, \mathrm{el}}\right\rangle \approx \sum_{j=1, N_{\mathrm{el}}} \gamma_{j, \mathrm{el}}^{i}\left|\Psi_{j, \mathrm{el}}^{0}\right\rangle,\left|\Psi_{i, \mathrm{ho}}\right\rangle \approx \\
\sum_{j=1, N_{\mathrm{ho}}} \gamma_{j, \mathrm{ho}}^{i}\left|\Psi_{j, \mathrm{ho}}^{0}\right\rangle .
\end{gathered}
$$

With $s, p, d$ and $f$ quantum dot shells included, dimensions (i.e. $N_{\text {el }}$ and $N_{\text {ho }}$ ) of Hamiltonians (9) and (10) are 10 (20 with spin), i.e. far smaller than the dimension of the unperturbed tight-binding problem $\left(10^{7}\right)$. Our approach has therefore a significant computational advantage especially for cases where numerous (e.g. parameter depended) external fields need to be calculated for the same nanostructure.

Once single particle energies and states are determined, the many-body states are calculated [23]. For the exact diagonalized wave functions, the Coulomb matrix elements are now given as

$$
V_{i j k l}=\sum_{a, b, c, d} \gamma_{a, i}^{*} \gamma_{b, j}^{*} \gamma_{c, k} \gamma_{d, l} V_{i j k l}^{0}
$$

where $V_{i j k l}^{0}$ for the zero-field case are defined as in Ref. [23]. The dipole matrix elements [23] are calculated accordingly.

\section{Results}

The effect of piezoelectricity is expected to be negligible for flat disk-shaped quantum dots [25], due to a small shear strain contribution. The piezoelectricity can typically be also neglected for low-strain nanosystems such as InAs/InP quantum dots [25]. On the other hand, the piezoelectricity is expected to play an important role for highly-strained pyramid or lens-shaped InAs/GaAs quantum dots, with pronounced shear strains occurring at curved top facets of quantum dots [27]. We therefore illustrate our theory on the example of lens-shaped InAs/GaAs quantum dot located on a one lattice constant thick InAs wetting layer. The height of the quantum dot is chosen as $3.5 \mathrm{~nm}$ and the base diameter is $25 \mathrm{~nm}$. This particular quantum dot constitutes a standard [14, 23] system allowing for a comparison of results obtained using different computational approaches. 
TABLE

Electron, hole and exciton $\left(E_{\mathrm{X}}\right)$ ground $(s)$ state energies, and splittings between electron and hole levels calculated by a full tight-binding (full TB) diagonalization and by an exact diagonalization approach. The effect of piezoelectricity is either neglected (no piezo.), or included at a different level of theory (linear only, quadratic only, linear and quadratic combined) with either DFT [19] or empirical parameters [15] used in the computation.

\begin{tabular}{|c|c|c|c|c|c|c|c|c|c|}
\hline \multirow{3}{*}{$\begin{array}{c}\mathrm{I} \\
\text { Energy } \\
{[\mathrm{meV}]}\end{array}$} & \multirow{3}{*}{$\begin{array}{c}\text { No } \\
\text { piezo. } \\
\text { Full TB }\end{array}$} & \multicolumn{2}{|c|}{ Linear } & \multicolumn{2}{|c|}{ Quadratic } & \multicolumn{2}{|c|}{ Linear and quadratic } & \multicolumn{2}{|c|}{ Linear } \\
\hline & & \multicolumn{6}{|c|}{ DFT parameters } & \multicolumn{2}{|c|}{ Empirical parameters } \\
\hline & & Full TB & Exact diag. & Full TB & Exact diag. & Full TB & Exact diag. & Full TB & Exact diag. \\
\hline$s$ & 1162.83 & 1165.30 & 1165.30 & 1162.73 & 1162.74 & 1165.40 & 1165.41 & 1164.56 & 1164.57 \\
\hline$p_{1}-s$ & 59.886 & 58.457 & 58.471 & 57.284 & 57.283 & 60.781 & 60.788 & 60.858 & 60.864 \\
\hline$\stackrel{0}{0} p_{2}-p_{1}$ & 3.049 & 5.496 & 5.501 & 7.883 & 7.889 & 0.666 & 0.666 & 0.850 & 0.853 \\
\hline$d_{1}-p_{2}$ & 57.688 & 55.550 & 55.963 & 51.914 & 52.039 & 59.691 & 59.779 & 59.724 & 59.823 \\
\hline$d_{2}-d_{1}$ & 3.172 & 3.910 & 3.819 & 6.342 & 6.324 & 2.087 & 2.054 & 2.136 & 2.101 \\
\hline$d_{3}-d_{2}$ & 5.377 & 6.094 & 6.052 & 8.742 & 8.736 & 4.302 & 4.301 & 4.230 & 4.228 \\
\hline$f_{1}-d_{3}$ & 52.243 & 51.366 & 51.674 & 45.122 & 45.186 & 54.664 & 54.727 & 54.901 & 54.971 \\
\hline \multirow{8}{*}{ 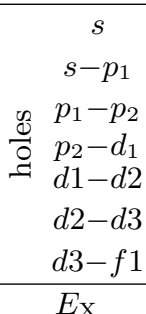 } & 266.42 & 271.90 & 271.71 & 267.36 & 267.31 & 269.20 & 269.18 & 268.72 & 268.70 \\
\hline & 9.049 & 6.452 & 6.717 & 7.253 & 7.305 & 9.090 & 9.115 & 8.648 & 8.692 \\
\hline & 8.888 & 13.143 & 13.455 & 11.371 & 11.418 & 9.851 & 9.852 & 10.137 & 10.114 \\
\hline & 1.243 & 1.475 & 1.191 & 1.604 & 1.538 & 1.252 & 1.277 & 0.836 & 0.886 \\
\hline & 10.061 & 9.693 & 12.100 & 9.238 & 10.266 & 10.428 & 11.015 & 10.296 & 10.981 \\
\hline & 4.849 & 1.971 & 2.036 & 2.882 & 2.614 & 3.970 & 3.961 & 4.149 & 4.095 \\
\hline & 2.212 & 5.944 & 7.217 & 5.924 & 6.466 & 2.841 & 2.867 & 3.128 & 3.122 \\
\hline & 874.07 & 871.69 & 871.60 & 873.25 & 873.85 & 873.55 & 873.70 & 873.39 & 873.37 \\
\hline
\end{tabular}

Table I shows electronic structure of several lowest electron and hole levels calculated using different level of approximation. In general, results obtained with the exact diagonalization method are in an excellent agreement with the full tight-binding method. For example, the electron $p$-shell splitting calculated using the exact diagonalization approach typically varies within $1 \%$ between both approaches. Similar accuracy is obtained for the energy of the electron ground $(s)$ state and splittings between higher electron states (e.g. $\left.d_{3}-d_{2}\right)$. A very good agreement is also apparent for the hole states, and the excitonic ground state energy $\left(E_{\mathrm{X}}\right)$. Moreover, the agreement between the full treatment and the approximate method holds well for all considered cases.

A further discussion is needed for comparison of results obtained with different piezoelectricity approaches. Table I shows that in agreement with Ref. [17] in the non-linear (DFT parametrized [19]) model one cannot simply neglect the quadratic term and account for the linear term only. It would lead to a significant overestimation of the electronic $p$-shell splitting $(5.5 \mathrm{meV})$ as compared to approach including both terms $(0.7 \mathrm{meV})$. However we note that results obtained using the linear theory, but with empirical piezoelectric tensors [15], give a reasonable level of accuracy (the electronic $p$-shell splitting is $0.85 \mathrm{meV}$ ), without the necessity of inclusion of quadratic terms. In other words, the linear theory with empirical parameters gives results close to those obtained a non-linear treatment with DFT derived parameters. In fact, as shown in Fig. 1, the spatial distribution of the piezoelectric potential reported by both

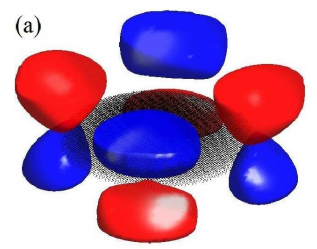

(c)

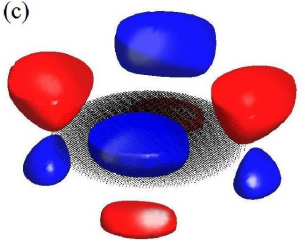

(b)

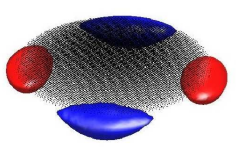

(d)

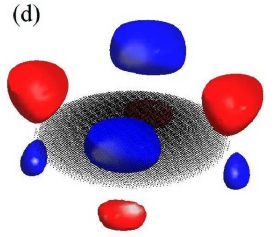

Fig. 1. Piezoelectric potential spatial distribution: (a) linear term included only (DFT parameters [19]), (b) quadratic term only (DFT parameters), (c) both linear and quadratic terms included (DFT parameters), (d) linear term included only (empirical parameters [15]). For (a), (c) and (d) cases isosurfaces were obtained for the value of the potential equal to $|30 \mathrm{mV}|$, whereas for (b) $|15 \mathrm{mV}|$ was used. Blue color corresponds to negative values of the potential, whereas red color points to positive values. Atoms constituting the quantum dot are represented as black points. The InAs wetting later and the GaAs barrier are not shown.

(linear and non-linear) approaches is qualitatively similar. It should be noted that most of the piezoelectric potential (and therefore the difference between both methods) arises in the GaAs matrix area, due to much larger GaAs piezoelectric modulus. Therefore the piezoelectric potential builds up predominately outside of the dot area, 
which effectively reduces impact of piezoelectricity on confined quantum dot states. Finally, we note that in a lens-shape quantum dot hole states do not form shellstructure, but there are pronounced $(\approx 10 \mathrm{meV})$ intershell splittings. Therefore the effect of piezoelectricity $(\approx 1-2 \mathrm{meV})$ on hole states is rather small and can be to a large degree neglected.

\section{Summary}

We present an exact diagonalization approach that allows for efficient and accurate calculation of excitonic properties of nanostructures under electric field. We illustrate our method with the example of piezoelectricity in a lens-shaped InAs/GaAs quantum dot. We found that the linear piezoelectricity theory with empirical parameters gives results similar to a non-linear theory using DFT parametrization. Therefore we conclude that the accuracy of piezoelectricity calculation is significantly affected by both the choice of the computational method and its parametrization.

\section{Acknowledgments}

This work was supported by the Polish Ministry of Science and Higher Education as a research project No. IP 2012064572 (Iuventus Plus).

\section{References}

[1] L. Jacak, P. Hawrylak, A. Wojs, Quantum Dots, Springer, Berlin 1998.

[2] D. Bimberg, M. Grundmann, N.N. Ledentsov, Quantum Dot Heterostructures, Wiley, New York 1998.

[3] D. Dalacu, K. Mnaymneh, X. Wu, J. Lapointe, G.C. Aers, P.J. Poole, R.L. Williams, Appl. Phys. Lett. 98, 251101 (2011).

[4] N. Gisin, G. Ribordy, W. Tittel, H. Zbinden, Rev. Mod. Phys. 74, 145 (2002).

[5] J. Chen, J.B. Altepeter, M. Medic, K.F. Lee, B. Gokden, R. H. Hadfield, S. W. Nam, P. Kumar, Phys. Rev. Lett. 100, 133603 (2008).

[6] O. Benson, C. Santori, M. Pelton, Y. Yamamoto, Phys. Rev. Lett. 84, 2513 (2000).
[7] M. Bayer, G. Ortner, O. Stern, A. Kuther, A.A. Gorbunov, A. Forchel, P. Hawrylak, S. Fafard, K. Hinzer, T.L. Reinecke, S.N. Walck, J.P. Reithmaier, F. Klopf, F. Schäfer, Phys. Rev. B 65, 195315 (2002).

[8] R. Stevenson, C.L. Salter, I. Farrer, C.A. Nicoll, D.A. Ritchie, A.J. Shields, Nature 439, 179 (2006).

[9] S. Seidl, M. Kroner, A. Högele, K. Karrai, R. Warburton, A. Badolato, P. Petroff, Appl. Phys. Lett. 88, 203113 (2006).

[10] M.B. Bavinck, M. Zielinski, B. Witek, T. Zehender, E. Bakkers, V. Zwiller, Nano Lett. 12, 6202 (2012).

[11] G.W. Bryant, M. Zielinski, N. Malkova, J. Sims, W. Jaskolski, J. Aizpurua, Phys. Rev. Lett. 105, 067404 (2010).

[12] K. Kowalik, O. Krebs, A. Lemaître, S. Laurent, P. Senellart, P. Voisin, J.A. Gaj, Appl. Phys. Lett. 86, 041907 (2005).

[13] A.J. Bennett, M.A. Pooley, R.M. Stevenson, M.B. Ward, R.B. Patel, A. Boyer de la Giroday, N. Sköld, I. Farrer, C.A. Nicoll, D.A. Ritchie, A.J. Shields, Nature Phys. 6, 947 (2010).

[14] M. Zielinski, Phys. Rev. B 86, 115424 (2012).

[15] O. Stier, M. Grundmann, D. Bimberg, Phys. Rev. B 59, 5688 (1999).

[16] M. Grundmann, O. Stier, D. Bimberg, Phys. Rev. B 52, 11969 (1995)

[17] G. Bester, Xifan Wu, D. Vanderbilt, A. Zunger, Phys. Rev. Lett. 96, 187602 (2006).

[18] G. Bester, A. Zunger, Xifan Wu, D. Vanderbilt, Phys. Rev. B 74, 081305(R) (2006).

[19] A. Beya-Wakata, P.-Y. Prodhomme, G. Bester, Phys. Rev. B 84, 195207 (2011).

[20] P.N. Keating, Phys. Rev. 145, 637 (1966).

[21] J.M. Jancu, R. Scholz, F. Beltram, F. Bassani, Phys. Rev. B 57, 6493 (1998).

[22] D.J. Chadi, Phys. Rev. B 16, 790 (1977).

[23] M. Zielinski, M. Korkusinski, P. Hawrylak, Phys. Rev. B 81, 085301 (2010).

[24] S. Schulz, S. Schumacher, G. Czycholl, Phys. Rev. B 73, 245327 (2006).

[25] G. Bester, A. Zunger, Phys. Rev. B 71, 045318 (2005).

[26] M. Gong, W. Zhang, G.C. Guo, L. He, Appl. Phys. Lett. 99, 231106 (2011).

[27] A. Schliwa, M. Winkelnkemper, D. Bimberg, Phys. Rev. B 76, 205324 (2007). 